\title{
Enhancement of collisionless shock ion acceleration by electrostatic ion two-stream instability in the upstream plasma
}

\author{
Rajesh Kumar, ${ }^{1, *}$ Youichi Sakawa, ${ }^{2}$ Leonard N. K. Döhl, ${ }^{3}$ Nigel Woolsey, ${ }^{3}$ and Alessio Morace ${ }^{2}$ \\ ${ }^{1}$ Graduate School of Science, Osaka University, Toyonaka, Osaka 560-0043, Japan \\ ${ }^{2}$ Institute of Laser Engineering, Osaka University, Suita, Osaka 565-0871, Japan \\ ${ }^{3}$ Department of Physics, University of York, Heslington, York YO10-5DD, United Kingdom
}

(Received 1 October 2018; published 5 April 2019)

\begin{abstract}
Ion acceleration in electrostatic collisionless shocks is driven by the interaction of the high-power laser with specially tailored near-relativistic critical density plasma. 2D EPOCH particle-in-cell simulations show that the ion acceleration is dependent on the target material used. In materials with low charge-tomass ratio $\langle Z / A\rangle$, proton beams with high flux and low energy spread are generated. In multi-ion plasmas the ions with different $\langle Z / A\rangle$ acquire different velocities under a non-oscillating component of electrostatic field in the upstream region. This relative drift between the protons $(\langle Z / A\rangle=1)$ and the lower $\langle Z / A\rangle$ ions leads to the excitation of electrostatic ion two-stream instability. This in turn generates a low-velocity component in the upstream expanding protons. The velocity distribution of the upstream expanding protons is further broadened toward the higher velocity by the electrostatic ion two-stream instability between reflected protons, which results in large number of protons being accelerated by the shock.
\end{abstract}

DOI: 10.1103/PhysRevAccelBeams.22.043401

\section{INTRODUCTION}

The development of high-intensity laser systems has opened a new era for laser-driven ions acceleration and there are several promising mechanisms for laser-driven ion acceleration. The energetic ion beams driven by laserplasma interaction have many potential applications such as accelerator physics, cancer therapy, proton radiography, and inertial confinement fusion [1-3]. However, producing a proton beam with high energy, high flux, and low energyspread proved to be the major challenge for practical applications [4,5]. Several mechanisms have been studied for laser-driven acceleration. At present, the most widely understood mechanism is Target Normal Sheath Acceleration (TNSA). This mechanism [6] and a related radiation-pressure hybrid-scheme [7] can drive protons to energies approaching $100 \mathrm{MeV}$. Alternative acceleration schemes, such as radiation-pressure acceleration that can achieve monoenergetic and higher energy suitable for applications impose strict and challenging conditions in experiments, such as the target thickness and laser contrast [8-10]. We describe a method to circumvent such constraints whilst providing methods to achieve higher ion energies.

*rajesh-dhiman@ile.osaka-u.ac.jp

Published by the American Physical Society under the terms of the Creative Commons Attribution 4.0 International license. Further distribution of this work must maintain attribution to the author(s) and the published article's title, journal citation, and DOI.
Collisionless shock acceleration (CSA) has been proposed separately by Denavit [11] and Silva [12], with a detailed theoretical investigation complemented by Fiuza [13]. These studies suggest that a special near-critical density profile, $N_{\mathrm{cr}}$, is important in order to control the sheath electric field, $E_{\mathrm{TNSA}}$, at the plasma-vacuum interface. This in turn affects the ion spectrum in CSA. The $E_{\text {TNSA }}$ amplitude can be reduced by using an exponentially decreasing density profile on the rear-side of the target [14], finally resulting in a quasimonoenergetic distribution for the CSA ions. CSA experiments using a linearly polarized $\mathrm{CO}_{2}$ laser with near- $N_{\text {cr }}$ gas-jet targets produced $20 \mathrm{MeV}$ proton beam [15]. A number of experiments have been carried out over the last few years to understand and characterize ion acceleration via CSA [16-21]. One aspect of CSA that is currently not well understood for accelerating mono-energetic ions to high energies is the effect of the target material used.

In this paper we used the EPOCH particle-in-cell (PIC) open source code [22] to study the generation of electrostatic (ES) collisionless shocks and proton acceleration from shocks formed in plasmas of different material composition. Our results show that a low average charge-to-mass ratio $(\langle Z / A\rangle)$ plasma produces a higher proton beam flux with a higher laser-to-proton energy conversion efficiency, and these differences become smaller at higher laser intensities. In the plasma with multi-ion species the ions with lower $\langle Z / A\rangle$ compared to the protons (with $\langle Z / A\rangle=1$ ) gain different velocities. This results in difference between relative drift velocities of the protons and lower $\langle Z / A\rangle$ ions in the upstream region of the shock. This leads to the 
excitation of an electrostatic ion two-stream instability (EITI) [23], which in turn enhances the number of the shockaccelerated protons [24]. To our best knowledge, most of the previous work has focused on shock formation [25-27] and ion heating [28-30]. This paper is the first investigation on the material (or $\langle Z / A\rangle$ ) dependence of EITI on CSA.

\section{SIMULATIONS}

We used 2D EPOCH simulations to investigate proton acceleration via CSA mechanism by modeling plasmas with different multi-ion compositions used commonly in experiments as target materials. The simulation box is $300 \times 6 \mu \mathrm{m}$ in size and composed of $9000 \times 180$ cells along the $x$ - and $y$-axis respectively. Each cell contains 24-30 particles depending upon the material being studied. Open and periodic boundary conditions are used along the $x$ - and $y$-axis respectively for both fields and particles.

The laser pulse and target in the PIC simulations use the parameters achievable at the Institute of Laser Engineering, Osaka University. An experiment would use the high intensity LFEX for the main interaction and the Gekko XII laser to preionize the target rear surface and create the necessary density profiles for CSA mechanism. The high intensity beam irradiated on the target front surface is linearly p-polarized with a temporal Gaussian profile of $1.5 \mathrm{ps}$ full-width at half-maximum and peak intensity of $1.4 \times 10^{19} \mathrm{~W} / \mathrm{cm}^{2}\left(a_{0}=3.35\right)$. The target consists of a tailored density profile increasing exponentially for $30 \mu \mathrm{m}$ with $5 \mu \mathrm{m}$ density scale length at $N_{\text {cr }}$ on laser side, a $5 \mu \mathrm{m}$ region of constant density at the relativistic critical density $a_{0} N_{\text {cr }}$, and an exponentially decreasing density with $30 \mu \mathrm{m}$ scale-length on the rear extending for $125 \mu \mathrm{m}$. The initial density profile is shown in Fig. 1(a), $t=0$. The corresponding ion densities are configured to ensure quasineutrality. The density profile and laser parameters are the same for all materials. The different material compositions and associated $\langle Z / A\rangle$ used in this study are summarized in Table I. The simulations are timed to reach peak irradiance at $1.5 \mathrm{ps}$ and have an initial electron and ion temperature of $T_{e}=T_{i}=500 \mathrm{eV}$.

\section{A. Collisionless shock formation}

Figure 1(a) shows the temporal evolution of $N_{H} / N_{\mathrm{cr}}$ in $\mathrm{C}_{2} \mathrm{H}_{3} \mathrm{Cl}$ for a number of times after the peak of the laser pulse, which enter the particle grid at $1.5 \mathrm{ps}$. During the time from $t=0$ to $t=1.75 \mathrm{ps}$ the laser interaction with plasma results in uniform electron heating via $J \times B$ [31]. This compresses the plasma in front of the exponentially decreasing density on the rear target side, as shown by the peak in the density profile in Fig. 1(a). As time evolves, the density on the target rear side is expanding, resulting in the formation of the ES collisionless shock. The shock propagates at velocity $V_{s h}$ in the forward direction. The exponentially decreasing density profile in the upstream
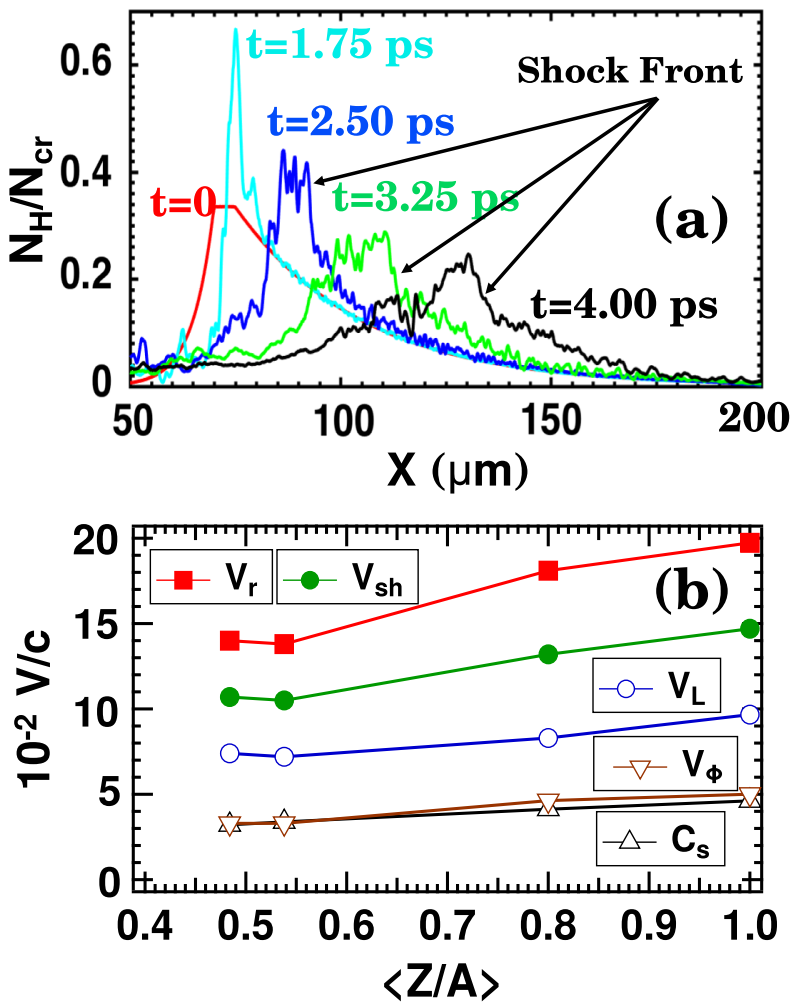

FIG. 1. (a) The temporal evolution of $N_{H} / N_{\text {cr }}$ in $\mathrm{C}_{2} \mathrm{H}_{3} \mathrm{Cl}$ at $t=0,1.75,2.50,3.25$, and 4.00 ps. (b) $\langle Z / A\rangle$ dependence of the ion acoustic speed $C_{s}$, the lower threshold $V_{L}$, the shock speed $V_{s h}, V_{\phi}=\sqrt{2 e \phi / m_{p}}$, and the velocity of the shock reflected protons $V_{r}$ at $t=4.0 \mathrm{ps}$.

plasma, the region ahead of the shock results in a uniform ES sheath field ahead of the shock, $E_{\mathrm{TNSA}}=T_{e} / e L_{g}$, where $T_{e}$ is the upstream electron temperature, $e$ is the electric charge, and $L_{g}$ is the exponentially decreasing rearside scale-length [14]. This $E_{\mathrm{TNSA}}$ field gives a uniform velocity $V_{0}$ to the upstream expanding protons.

For an ES collisionless shock to accelerate protons by ion reflection the ES potential $\phi$ at the shock must satisfy the relation $Z e \phi \geq \frac{1}{2} A m_{p}\left(V_{s h}-V_{0}\right)^{2}$, where $Z=A=1$ for protons, and $m_{p}$ is the proton mass [32]. Shock reflected protons have a velocity $V_{r}=2 V_{s h}-V_{0} . \phi$ can be estimated by subtracting the non-oscillating component of $E_{\mathrm{TNSA}}$ from the overall ES field $\left(E_{x}\right)$ and integrating along the $x$-axis, $\phi=\int_{\infty}^{x}\left(E_{x}-E_{\mathrm{TNSA}}\right) d x$. At $t=2.5 \mathrm{ps}, \phi$ is large, as a result, acceleration of upstream protons via

TABLE I. The charge-to-mass ratio for different targets. Note, all ions are fully ionized except chlorine which has $Z_{\mathrm{Cl}}=15$.

\begin{tabular}{lcccc}
\hline \hline Material & $\mathrm{C}_{2} \mathrm{H}_{3} \mathrm{Cl}$ & $\mathrm{CH}$ & $\mathrm{He}^{3} \mathrm{H}$ & Hydrogen \\
\hline$\langle Z / A\rangle$ & 0.4839 & 0.5385 & 0.8 & 1 \\
$\langle Z\rangle$ & 30 & 7 & 4 & 1 \\
$\langle A\rangle$ & 62 & 13 & 5 & 1 \\
\hline \hline
\end{tabular}


shock reflection occurs. By $t=4.0 \mathrm{ps}, \phi$ is gradually dissipating and reflection of protons becomes negligible.

Figure 1(b) compares the $\langle Z / A\rangle$ dependence of a number of velocities relative to the speed of light, c. Here $V_{\phi}=$ $\sqrt{2 e \phi / m_{p}}$ and $V_{L}=V_{s h}-V_{\phi}$ is the lower threshold for the proton reflection. The velocities $V_{r}, V_{s h}, V_{L}, V_{\phi}$ and the ion acoustic speed $C_{S}$ show a general increase with $\langle Z / A\rangle$. The increasing velocities result from a $\sqrt{Z / A}$ dependence in the hole-boring velocity $V_{H B}=c \sqrt{\frac{Z}{2 A} \frac{m_{e}}{m_{p}} \frac{N_{\text {cr }}}{N_{e}} a_{0}^{2}}$ with the nonrelativistic limit [33]. This is a good approximation for the low $a_{0}$ used. Here $m_{e}$ is the electron mass, $a_{0}$ and $N_{e}$ are the same for all the materials. The shock front moves forward at velocity $V_{s h}$, which is powered by the laserdriven hole boring process at the target front. As there is a rear surface density drop, $V_{s h}$ increases in time. Protons with velocities $V_{0}$ that lie between $V_{L}$ and $V_{s h}$ are accelerated via shock reflection.

A 2D-relativistic Maxwellian $f(E) \propto E \exp \left(-E / T_{e}\right)$ is used for the electron energy spectrum in the upstream region. For all materials, $T_{e} \sim 2.0 \mathrm{MeV}$. This implies that the ion acoustic speed is given by $C_{s}=\sqrt{Z T_{e} / A m_{p}}$ is only a function of $\langle Z / A\rangle$ as shown in Fig. 1(b).

Figures 2(a)-2(d) show the proton phase-space at $4.0 \mathrm{ps}$ for each of the materials shown in Table I. The red solid line overlaid on each of the plots corresponds to the proton velocity distribution in the upstream region ahead of the shock front. This is integrated from the velocities within the vertical box in the phase-space plots, which spans across a range of $\Delta x=3 \mu \mathrm{m}$. The horizontal lines are the material dependent velocities $V_{r}, V_{s h}$, and $V_{L}$. Protons that satisfy the inequality $V_{L} \leq V_{0} \leq V_{s h}$ are shock reflected. For target materials with lower $\langle Z / A\rangle$, such as $\mathrm{C}_{2} \mathrm{H}_{3} \mathrm{Cl}$ and $\mathrm{CH}$, a significantly larger number of protons satisfy this inequality when compared to $\mathrm{He}^{3} \mathrm{H}$ and $\mathrm{H}$. As a result, a larger number of protons are shock accelerated. This is deduced by comparing Figs. 2(a) and 2(b) with Figs. 2(c) and $2(\mathrm{~d})$. The proton velocity distribution is broader at lower $\langle Z / A\rangle$ due to the presence of the lower $\langle Z / A\rangle$ ions.

A broad velocity distribution results in a variation of the Mach number (M). To account for this we define $M=\left(V_{s h}-V_{\text {mean }}\right) / C_{s}$, where $V_{\text {mean }}$ is a mean value of $V_{0}$ in the distribution. In our calculations, we obtain $M=$ 1.6-1.7 for all materials. This is in agreement with the critical Mach number $\left(M_{\mathrm{cr}}=1.6\right)$, which has been previously validated in PIC simulations for linearly polarized laser pulse $[11,13]$. Therefore, the particle acceleration, and thus shock dissipation, is expected when the ions are being reflected. The maximum velocity of the reflected protons from the shock front is expected to reach $2 V_{s h}-V_{L}$. Simulations show that the velocity distribution just ahead of the shock peaks around this maximum velocity. In the upstream plasma, protons with higher $\langle Z / A\rangle$ are preferentially accelerated in the $x$-direction by the ES field $E_{\mathrm{TNSA}}$, which in turn drives the EITI.
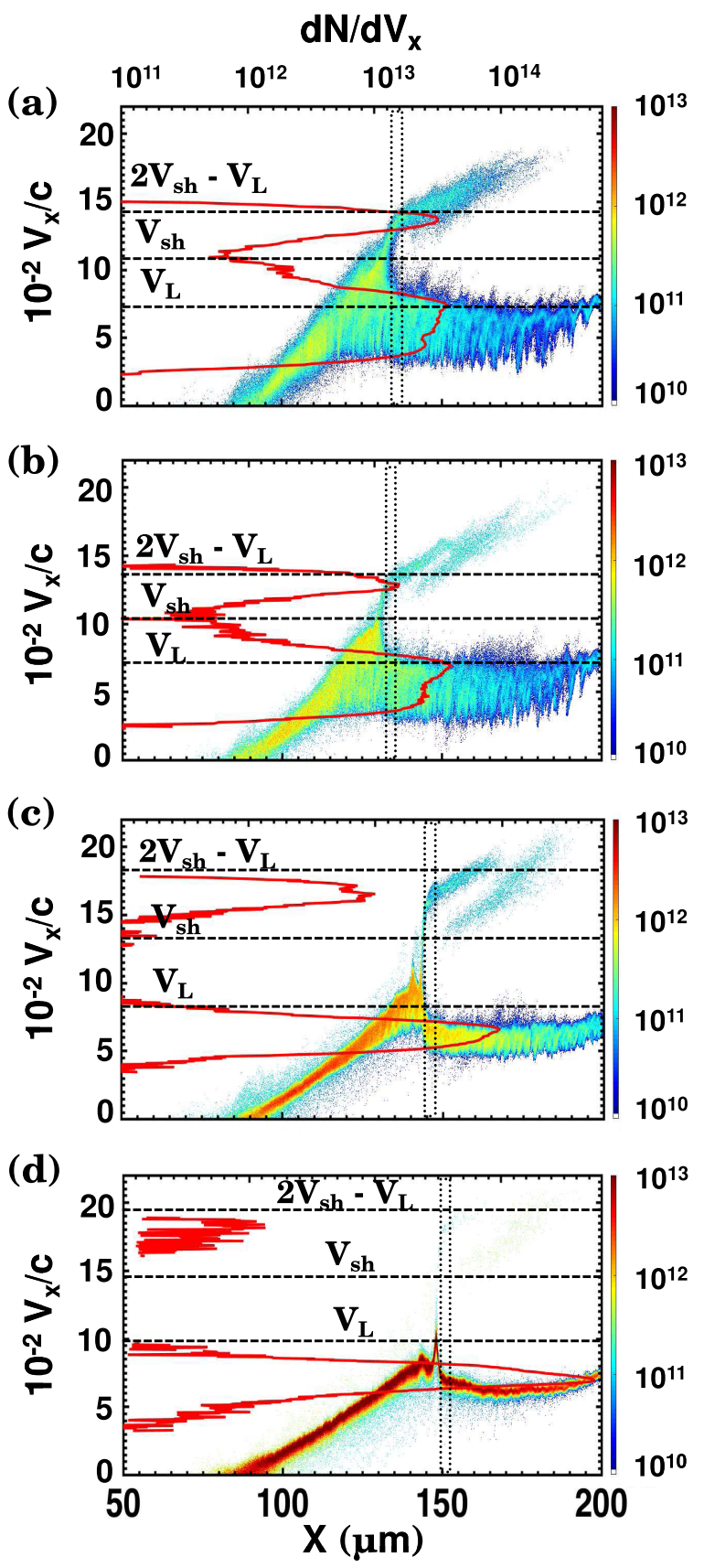

FIG. 2. A phase-space and velocity spectrum of the protons taken at $\Delta x=3 \mu \mathrm{m}$ in the upstream of the shock front at $t=4.0$ ps are shown for (a) $\mathrm{C}_{2} \mathrm{H}_{3} \mathrm{Cl}$, (b) $\mathrm{CH}$, (c) $\mathrm{He}^{3} \mathrm{H}$, and (d) Hydrogen.

Figure 3(a) shows the energy distribution of accelerated protons in the whole upstream region. We find that the energy spread increases with increasing $\langle Z / A\rangle$. Simulations show that this occurs at early times when $\phi$ is relatively weak. This is due to hole-boring on the front side of the target. These protons have a different velocity distribution and lower energies compared to the shock reflected protons, which results in the larger energy spread. The differences are apparent in Figs. 2(c) and 2(d). 

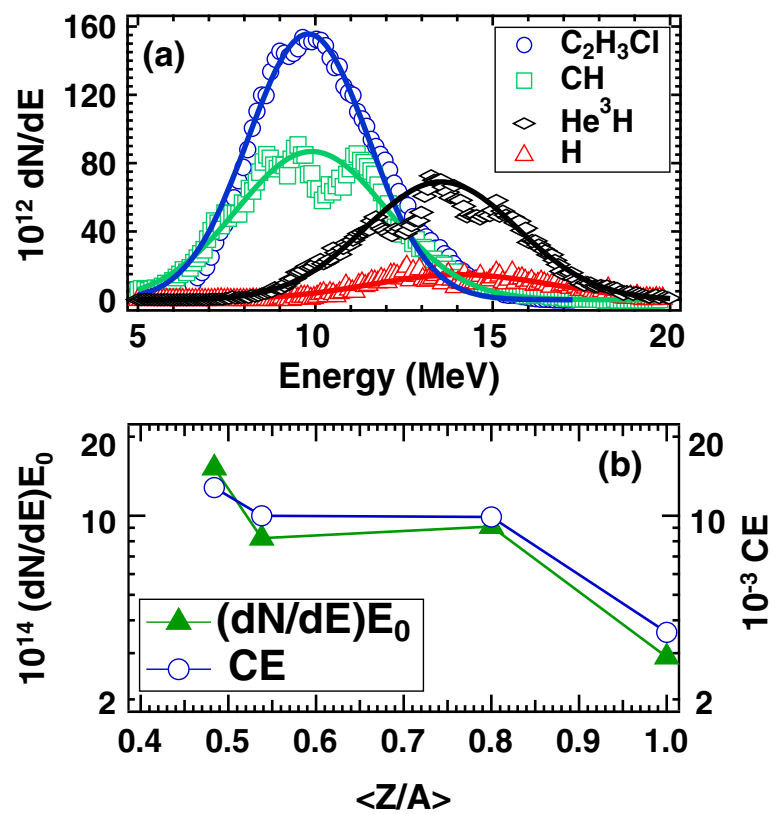

FIG. 3. (a) The energy spectra of the reflected protons in $\mathrm{C}_{2} \mathrm{H}_{3} \mathrm{Cl}$ (circles), $\mathrm{CH}$ (rectangles), $\mathrm{He}^{3} \mathrm{H}$ (diamonds) and, $\mathrm{H}$ (triangles). (b) $\langle Z / A\rangle$ dependencies of the $(d N / d E) E_{0}$ and laser to the reflected protons conversion efficiency (CE) taken in the whole upstream region at $t=4.0 \mathrm{ps}$.

The energy distribution of the shock reflected protons multiplied by the peak energy in the distribution, $E_{0}$, is shown as a function of $\langle Z / A\rangle$ in Fig. 3(b). The laser-toproton energy conversion efficiency $(\mathrm{CE})$ is compared to the distribution in the same figure. Material with the lowest $\langle Z / A\rangle\left(\mathrm{C}_{2} \mathrm{H}_{3} \mathrm{Cl}\right)$ show a $\mathrm{CE}$ almost an order of magnitude higher than a pure proton target with the highest $\langle Z / A\rangle$. In comparison, the peak energy of the distributions is increasing by a comparatively small factor of $\sim 1.4$ from $\mathrm{C}_{2} \mathrm{H}_{3} \mathrm{Cl}$ to $\mathrm{H}$ as shown in Fig. 3(a).

Figures 4(a) and 4(b) show the $x-y$ spatial profile of the ES field, $E_{x}$, for the $\mathrm{C}_{2} \mathrm{H}_{3} \mathrm{Cl}$ upstream plasma. The simulation box size is $y=6$ and $20 \mu \mathrm{m}$, respectively. Both cases show a wavelike structure propagating in the $x$-direction, with increasing wavelength for larger $x$. The $E_{x}$ profile along the $x$-axis is averaged along the $y$-axis and overlaid on the phase-space of the corresponding proton velocity distribution in the upstream shown in Fig 4(c). The modulation in $E_{x}$ shows comparative correlation with the proton phase-space distributions. These modulations are caused by the EITI in the upstream plasma. This is excited by the relative drift velocity $\left(v_{d}\right)$ between the two ion populations in plasmas with multi-ion species [23]. Our simulations show that this relative drift is driven by the $E_{\mathrm{TNSA}}$ in the upstream plasma which results in slower moving heavier ions with low $\langle Z / A\rangle$ and faster moving protons with high $\langle Z / A\rangle$. Simulations done by Zhang [26] have shown that in the transverse size-reduced simulation EITI propagates in the $x$-direction, whereas in the large transverse size simulation it propagates obliquely. In our
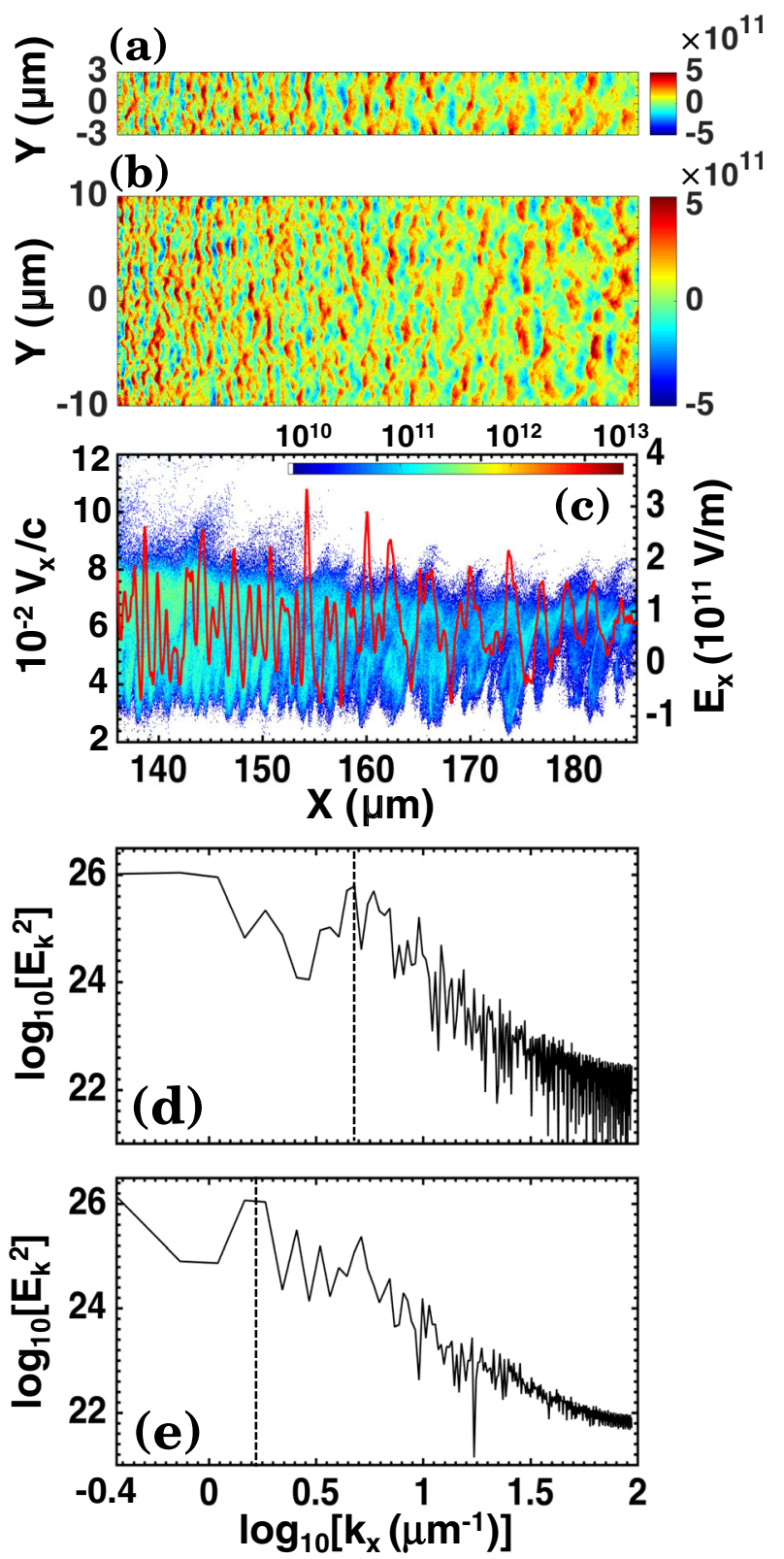

FIG. 4. 2D ES field $\left(E_{x}\right)$ distribution averaged over two lasercycles in the upstream region at $t=4.0 \mathrm{ps}$ in $\mathrm{C}_{2} \mathrm{H}_{3} \mathrm{Cl}$ for the simulations box size of (a) $y=6 \mu \mathrm{m}$ and (b) $y=20 \mu \mathrm{m}$. (c) Phase-space of the upstream expanding protons and 1D profile of $E_{x}$. Derived power spectrum of $E_{x}$ at $t=4.0$ ps taken in the region of (d) $x=135-145 \mu \mathrm{m}$ and (e) $x=165-175 \mu \mathrm{m}$.

case, since the propagation directions of EITI are in the $x$-direction both for the small and the large transversesize simulations [Figs. 4(a) and 4(b)], the instability is not oblique but longitudinal.

Temporal evolution of the upstream ion velocity distribution $d N / d V_{x}$ in the $\mathrm{C}_{2} \mathrm{H}_{3} \mathrm{Cl}$ plasma is compared in Figs. 5(a) and 5(b). These plots were captured just ahead of the shock at $t=2.0 \mathrm{ps}$ and $4.0 \mathrm{ps}$ respectively. Initially, $v_{d}$ is small and the instability has not established. As time 

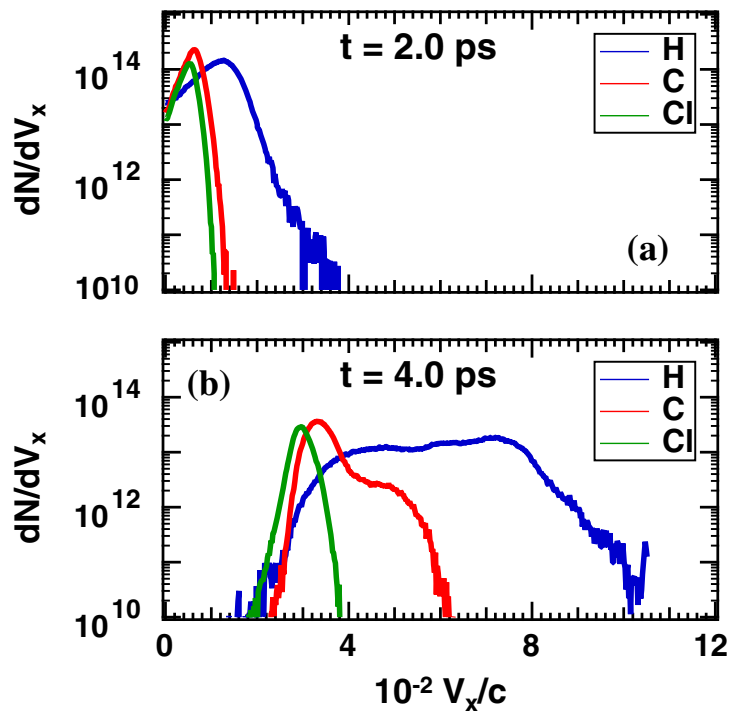

FIG. 5. Velocity spectrum of the expanding ions taken at $\Delta x=$ $3 \mu \mathrm{m}$ in the upstream region at (a) $t=2.0 \mathrm{ps}$ and (b) $t=4.0 \mathrm{ps}$ in $\mathrm{C}_{2} \mathrm{H}_{3} \mathrm{Cl}$.

evolves, $v_{d}$ increases, which leads to a growth in the instability. This results as shown in Fig. 5(b), in a highvelocity tail in the $\mathrm{C}$-ion and a low-velocity component to the proton distributions. We note that the chlorine distribution does not broaden in time. This observation suggests that the broadening of proton distributions discussed in relation to Fig. 2 results from the EITI.

Temporal evolution of the velocity at the peak of the $d N / d V_{x}$ distribution for Cl-ions, $V_{\mathrm{Cl}}$ (from the $\mathrm{C}_{2} \mathrm{H}_{3} \mathrm{Cl}$ target) is plotted in Fig. 6. $V_{\mathrm{Cl}}$ shows a logarithmic dependence with time indicating that $V_{\mathrm{Cl}}$ is determined by the upstream $E_{\mathrm{TNSA}}$. By taking a derivative of a fit to the $V_{\mathrm{Cl}}$ distribution with $\langle Z / A\rangle=0.429$, we infer that the time dependent $E_{\text {TNSA }}$ evolves with a $1 / t$ dependence as shown in Fig. 6. The temporal variations for $V_{\mathrm{Cl}}$ and $E_{\mathrm{TNSA}}$ relationship described by Mora [34]. The time dependence of the C-ion velocity $\left(V_{C}\right)$ at the peak of $d N / d V_{x}$ agrees well with calculations of $V_{C}$ based on the inferred $E_{\mathrm{TNSA}}$ and $\langle Z / A\rangle=0.5\left(V_{\mathrm{C}-\mathrm{TNSA}}\right)$. At $t=2.5 \mathrm{ps}$, a high-velocity tail $\left(V_{C}^{H}\right)$ appears in $V_{C}$. This results from acceleration by an electric field associated with the EITI. The calculated velocity for $\mathrm{H}$-ions from $E_{\mathrm{TNSA}}\left(V_{\mathrm{H}-\mathrm{TNSA}}\right)$ with $\langle Z / A\rangle=1$, which gives the maximum possible velocity for H-ions caused by $E_{\mathrm{TNSA}}$, is included for the comparison in Fig. 6 . We approximate the simulated velocity distributions for protons as a sum of the three 1D-shifted Maxwellian's. These velocities, the lower $\left(V_{H}^{L}\right)$, mean $\left(V_{H}^{M}\right)$, and higher $\left(V_{H}^{H}\right)$ velocity Maxwellian distributions are shown in Fig. 6. We note that $V_{C}^{H}$ and $V_{H}^{L}$ represent the EITI accelerated and decelerated C-ion and proton populations, respectively, and these populations have similar velocities. This occurs as an additional EITI develops due to the relative drift between the shock reflected proton population moving at approximately $2 V_{s h}-V_{0}$, through the upstream expanding proton

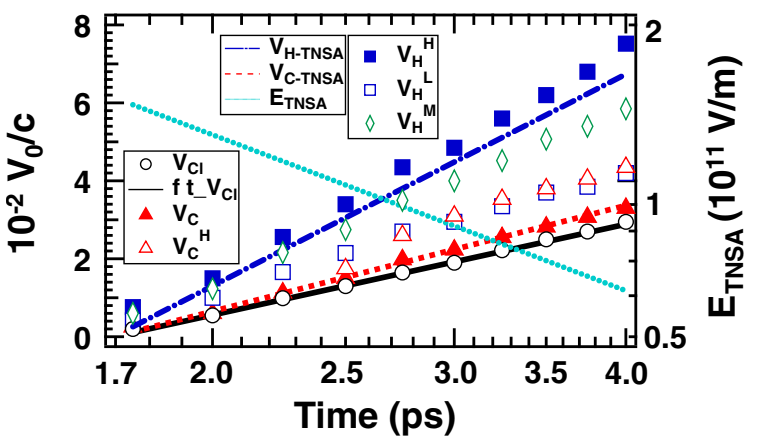

FIG. 6. Time evolution of the expanding ion speed in $\mathrm{C}_{2} \mathrm{H}_{3} \mathrm{Cl}$. The velocity of Cl-ions $V_{\mathrm{Cl}}$ (open circles) is derived from the peak of the velocity spectrum $\left(d N / d V_{\mathrm{Cl}}\right)$ taken at $\Delta x=3 \mu \mathrm{m}$ in the upstream region and fitted to logarithmic curve $V_{\mathrm{Cl}}$ (solid line). $E_{\mathrm{TNSA}}$ (dotted line) is derived from $V_{\mathrm{Cl}}$ and follows a $1 / t$ dependence. Temporal variation of $V_{C}$ (filled triangles) agrees well with the calculated $V_{C}\left(V_{\mathrm{C}-\mathrm{TNSA}}\right.$, dashed line) from $V_{\mathrm{Cl}} \cdot V_{C}^{H}$ (open triangles) shows the high-velocity tail of C-ions, which appears at $t=2.5 \mathrm{ps}$, and overlaps with the lower-velocity $\left(V_{H}^{L}\right)$ (open rectangles) component of $\mathrm{H}$-ions. $V_{H}^{H}$ (filled rectangles) represents the higher-velocity component of $\mathrm{H}$-ions, which is larger than the velocity calculated from $E_{\mathrm{TNSA}}\left[V_{\mathrm{H}-\mathrm{TNSA}}\right.$, (dotdashed line)].

population moving at $V_{0}$. This further increases the number of protons satisfying the condition for CSA.

\section{B. Electrostatic ion two-stream instability}

In the cold plasma approximation, the dispersion relation for EITI is given by [30]

$$
1+\frac{1}{k_{x}^{2} \lambda_{\mathrm{De}}^{2}}=\frac{\omega_{p 1}^{2}}{\omega^{2}}+\frac{\omega_{p 2}^{2}}{\left(\omega-k_{x} v_{d}\right)^{2}}
$$

where $k_{x}$ is the wave number in the $x$-direction, $\lambda_{\mathrm{De}}$ is the Debye length, $\omega_{p 1}$ and $\omega_{p 2}$ are the proton and heavy ion plasma frequencies, and $v_{d}$ is the relative drift between the two ion populations. In multi-ion plasmas, $\omega_{p 1}$ is the proton plasma frequency $\omega_{p p}$, and $\omega_{p 2}$ is the heavy-ion plasma frequency, as in, $\omega_{p 2}$ is the carbon plasma frequency $\omega_{p c}$ for $\mathrm{C}_{2} \mathrm{H}_{3} \mathrm{Cl}$ and $\mathrm{CH}$.

For $\mathrm{C}_{2} \mathrm{H}_{3} \mathrm{Cl}$ at $t=4.0 \mathrm{ps}$, the maximum amplitude of the modulation in $E_{x}$ in the region of $x=135-145 \mu$ m occurs at $k_{x}=4.7 \times 10^{6} \mathrm{~m}^{-1}$ [dashed line in Fig. 4(d))], and $\lambda_{\mathrm{De}}=$ $0.4 \mu \mathrm{m}$ (for $N_{e}=6.5 \times 10^{20} \mathrm{~cm}^{-3}$ and $T_{e}=2 \mathrm{MeV}$ ) at $x=140 \mu \mathrm{m}$ in the upstream region. This results in $k_{x} \lambda_{\mathrm{De}}>1$, enabling us to neglect the second term on the left-hand side of Eq. (1). At $x=140 \mu \mathrm{m}$, the carbon ion and proton number densities are $N_{C}=4 \times 10^{19} \mathrm{~cm}^{-3}$ and $N_{p}=1.3 \times 10^{20} \mathrm{~cm}^{-3}$ giving $\omega_{p c}=\omega_{p p}=1.5 \times 10^{13} \mathrm{sec}^{-1}$. Equation (1) has an unstable solution with the maximum growth rate of $\sim \omega_{p c} / 2$ at $k_{x} \sim \sqrt{3} \omega_{p c} / v_{d}$. We take $v_{d}=$ $V_{H}^{H}-V_{C}=0.042 \mathrm{c}$ at $t=4.0 \mathrm{ps}$. This gives, $k_{x} \sim 2.1 \times 10^{6} \mathrm{~m}^{-1}$, 
which agrees relatively well with the value of $k_{x}=4.7 \times$ $10^{6} \mathrm{~m}^{-1}$ extracted from simulation. We refer to this as heavy-ion EITI.

At $x=160 \mu \mathrm{m}, N_{C}=1 \times 10^{19} \mathrm{~cm}^{-3}$, which gives $\omega_{p c}=$ $7.2 \times 10^{12} \mathrm{sec}^{-1}$, and $v_{d}=0.040 \mathrm{c}$ at $t=4.0 \mathrm{ps}$. Thus, $k_{x} \sim 1.0 \times 10^{6} \mathrm{~m}^{-1}$, which is comparable to $k_{x} \sim 1.6 \times$ $10^{6} \mathrm{~m}^{-1}$ as shown in simulation in Fig. 4(e). Hence, it is clear from Figs. 4(d) and 4(e), $k_{x}$ decreases as $x$ increases because the plasma density decreases as shown in Fig. 1(a) $(t=4.0 \mathrm{ps})$.

At $t>2.5 \mathrm{ps}$, the number of protons reflected by the shock front increases, which contributes to the excitation of the EITI between the expanding and the reflected protons. This is referred to as reflected-proton EITI. This leads to the generation of a high-velocity tail in $V_{H}^{H}$ in the upstream plasma. In the case of reflected-proton EITI, $\omega_{p 1}=\omega_{p p}$ and $\omega_{p 2}$ is the reflected proton plasma fre-

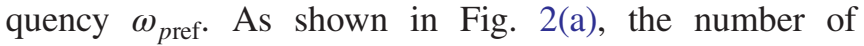
reflected protons is smaller than that of the expanding protons even at $t=4.0 \mathrm{ps}$. Hence, $\omega_{\text {pref }}<\omega_{p p}$. The maximum growth rate of reflected-proton EITI [30] is $\sim\left(3 \sqrt{3}\left(N_{\text {ref }} / N_{\text {exp }}\right) / 16\right)^{1 / 3} \omega_{p p}$ at $k_{x} \sim \omega_{p p} / v_{d}^{r}$. The relative drift between the reflected and expanding protons is $v_{d}^{r}=$ $0.064 \mathrm{c}$ at $x=140 \mu \mathrm{m}$ and $t=4.0 \mathrm{ps}$. Therefore, $k_{x} \sim 6.8 \times 10^{5} \mathrm{~m}^{-1}$, which is comparable to the $k_{x}$ value for the maximum growth rate of heavy-ion EITI at $x=$ $140 \mu \mathrm{m}$ by a factor of 0.31 . As a result, this suggests both that heavy-ion and reflected-proton EITIs occur with similar growth rates and wave numbers.

$V_{\mathrm{H}-\mathrm{TNSA}}$ or the maximum possible velocity of $\mathrm{H}$-ions accelerated by $E_{\mathrm{TNSA}}$ in $\mathrm{C}_{2} \mathrm{H}_{3} \mathrm{Cl}$ is shown in Fig. 6 . We infer that a large number of protons is accelerated by the wave electric field of reflected-proton EITI and obtain the expansion velocity $V_{0}$ larger than $V_{\mathrm{H}-\mathrm{TNSA}}$. This acceleration generates a significant number of expanding protons with the velocities larger than $V_{L}$, and results in the enhancement of the shock accelerated protons. Simulation studies done by Grassi et al. [35], have shown that the development of ion turbulence in $\mathrm{H}$ plasma leads to the heating of upstream ions, which allows a fraction of ions to exceed the threshold for ion reflection from the shock front.

To confirm the effect of $\langle Z / A\rangle$ on EITI, PIC-simulations with the same laser and density properties used throughout are compared to fully ionized carbon deuterium (CD) plasma. As shown in Fig. 7(a), since both $\mathrm{C}$ and D-ions have the same $\langle Z / A\rangle=0.5$, both ions are accelerated by $E_{\mathrm{TNSA}}$ in the upstream plasma, and there is no relative drift between them, as in, $v_{d}=0$. Therefore, no instability is excited, and no broadening of the upstream ions occurs. In multi-ion plasmas, a high-velocity tail and a low-velocity component appear in the smaller and larger $\langle Z / A\rangle$ ions, respectively. Figure 7(b) represents upstream ion velocity distribution for $\mathrm{CCl}_{2}$ plasma with fully ionized $\mathrm{C}$ and

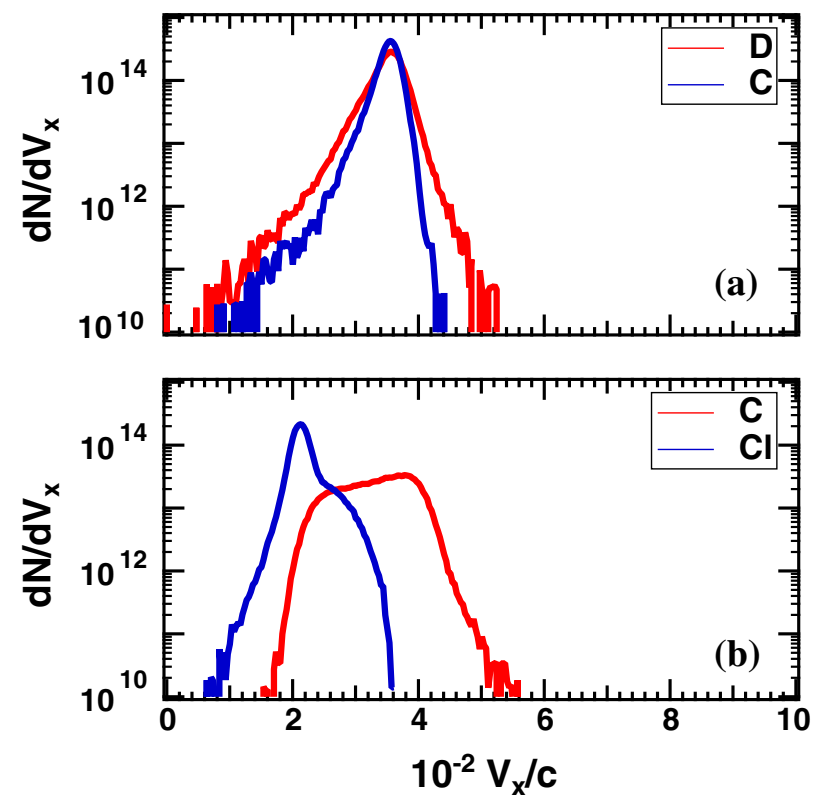

FIG. 7. Velocity spectrum of the expanding ions in (a) CD and (b) $\mathrm{CCl}_{2}$ targets taken at $\Delta x=3 \mu \mathrm{m}$ in the upstream region at $t=4.0 \mathrm{ps}$.

$Z_{\mathrm{Cl}}=10$. A high-velocity tail in Cl-ions and a low-velocity component of $\mathrm{C}$-ions appear because of heavy-ion EITI.

\section{DISCUSSION AND SUMMARY}

We further generalize our results by finding the dependence of the peak energy of the reflected protons and proton flux on the laser intensity corresponding to the $a_{0}=3.35$, 10 , and 33. In order to compensate for relativistically induced transparency, the initial electron density is varied

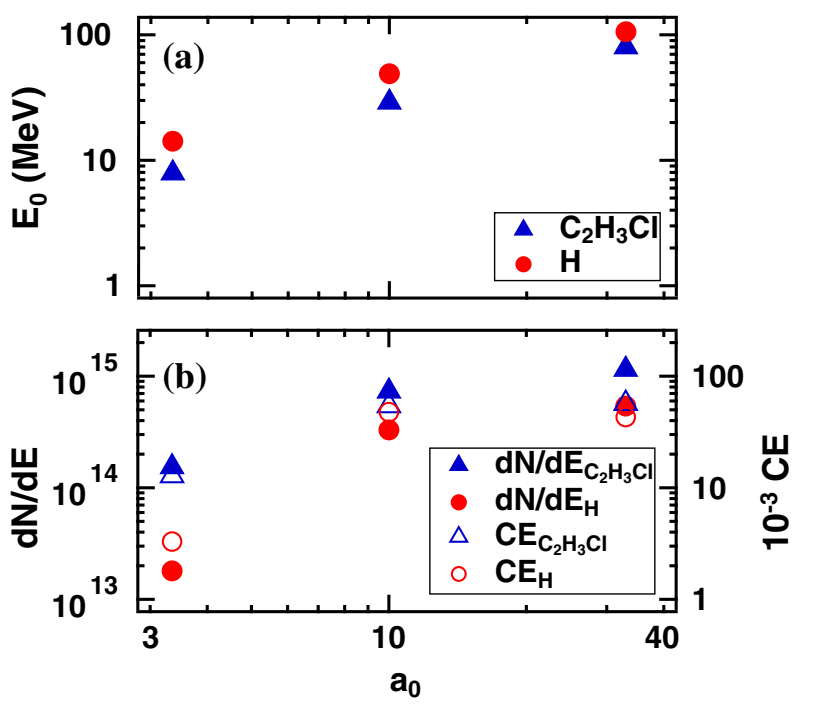

FIG. 8. (a) The peak energy $E_{0}$ of the shock reflected protons (b) the laser to proton conversion efficiency (CE) and the number of the shock accelerated protons $d N / d E$ at $E_{0}$ in $\mathrm{C}_{2} \mathrm{H}_{3} \mathrm{Cl}$ and $\mathrm{H}$ plasmas. 
for different laser intensities by $a_{0} N_{\text {cr }}$ for all plasmas. Figure 8 represents the peak energy $\left(E_{0}\right)$, conversion efficiency (CE), and number $\left(d N / d E\right.$ at $\left.E_{0}\right)$ of the shock accelerated protons for different laser intensities corresponding to $a_{0}=3.35,10$, and 33 in $\mathrm{C}_{2} \mathrm{H}_{3} \mathrm{Cl}$ and $\mathrm{H}$ plasmas. For $\mathrm{C}_{2} \mathrm{H}_{3} \mathrm{Cl}$ plasma, $E_{0}, d N / d E$, and $\mathrm{CE}$ increase with increasing laser intensity. However for $\mathrm{H}$ plasma, $E_{0}$ and $d N / d E$ increase but CE first increases and then decreases with increasing laser intensity. The $E_{0}$, $d N / d E$, and $\mathrm{CE}$ are always larger for $\mathrm{C}_{2} \mathrm{H}_{3} \mathrm{Cl}$ plasma compared to $\mathrm{H}$ plasma. As shown in Fig. 8(b), the increment in the $d N / d E$ and $\mathrm{CE}$ for the higher laser intensity is different from the lower laser intensity, whereas $d N / d E$ and $\mathrm{CE}$ of the shock accelerated protons are always higher in lower $\langle Z / A\rangle\left(\mathrm{C}_{2} \mathrm{H}_{3} \mathrm{Cl}\right)$ plasma than pure $\mathrm{H}$ plasma. At lower laser intensity $\left(a_{0}=3.35\right)$, these increments are found to be $\sim 8.6$ and $\sim 4$, while at higher laser intensity $\left(a_{0}=33\right)$, these increments are found to be $\sim 2$ and $\sim 1.3$.

We have performed the 2D PIC simulations in a plane wave approximation with an infinite spot-size. In the 3D scenario a smaller amplitude TNSA field will appear on the rear-side of the target according to Xiao et al. [36], which results in a quasimonoenergetic distribution for the CSA ions [14]. Even with this small amplitude TNSA field, the ions with different $\langle Z / A\rangle$ will be accelerated to the different velocities. This leads to the development of EITI in multiion species plasma.

In summary, we have presented a PIC simulation study of the ES collisionless shock proton acceleration in different target materials. Protons with high flux and narrower energy spread can be produced using the lower $\langle Z / A\rangle$ materials. The excitation of heavy-ion EITI leads to a large velocity spread in the upstream region of protons in multiion materials. The velocity distribution of the upstream expanding protons is further broadened toward the higher velocity by the EITI between reflected protons. As a result, a larger number of protons are driven by collisionless shock acceleration mechanism. This work supports future experimental work and investigations to a complete understanding of EITI and collisionless shocks using accessible experimental parameters.

\section{ACKNOWLEDGMENTS}

This research was partially supported by Japan Society for the Promotion of Science (JSPS) KAKENHI Grant No. $15 \mathrm{H} 02154$ and by funding from Engineering and Physical Science Research Council (EPSRC) grant [EP/ L01663X/1] and Science and Technology Facilities Council (STFC).

[1] H. Daido, M. Nishiuchi, and A. S. Pirozkhov, Review of laser-driven ion sources and their applications, Rep. Prog. Phys. 75, 056401 (2012).
[2] A. Macchi, M. Borghesi, and M. Passoni, Ion acceleration by superintense laser-plasma interaction, Rev. Mod. Phys. 85, 751 (2013).

[3] M. Roth, T. E. Cowan, M. H. Key, S. P. Hatchett, C. Brown, W. Fountain, J. Johnson, D. M. Pennington, R. A. Snavely, S. C. Wilks, K. Yasuike, H. Ruhl, P. Pegoraro, S. V. Bulanov, E. M. Campbell, M. D. Perry, and H. Powell, Fast Ignition by Intense Laser-Accelerated Proton Beams, Phys. Rev. Lett. 86, 436 (2001).

[4] S. V. Bulanov, T.Z. Esirkepov, V.S. Khororshkov, A. Z. Kuznetsov, and F. Pegoraro, Oncological hadrontherapy with laser ion accelerators, Phys. Lett. A 299, 240 (2002).

[5] U. Linz and J. Alonso, What will it take for laser driven proton accelerators to be applied to tumor therapy?, Phys. Rev. ST Accel. Beams 10, 094801 (2007).

[6] F. Wagner, O. Deppert, C. Brabetz, P. Fiala, A. Kleinschmidt, P. Poth, V. A. Schanz, A. Tebartz, B. Zielbauer, M. Roth, T. Stohlker, and V. Bagnoud, Maximum Proton Energy above $85 \mathrm{MeV}$ from the Relativistic Interaction of Laser Pulses with Micrometer Thick $\mathrm{CH}_{2}$ Targets, Phys. Rev. Lett. 116, 205002 (2016).

[7] A. Higginson, R. J. Gray, M. King, R. J. Dance, S. D. R. Williamson, N. M. H. Butler, R. Wilson, R. Capdessus, C. Armstrong, J. S. Green, S. J. Hawkes, P. Martin, W. Q. Wei, S. R. Mirfayzi, X. H. Yuan, S. Kar, M. Borghesi, R. J. Clarke, D. Neely, and P. McKenna, Near-100 MeV protons via a laser-driven transparency-enhanced hybrid acceleration scheme, Nat. Commun. 9, 724 (2018).

[8] I. J. Kim, K. H. Pae, W. Choi, C. L. Lee, H. T. Kim, H. Singhal, J. H. Sung, S. K. Lee, H. W. Lee, P. V. Nickles, T. M. Jeong, C. M. Kim, and C. H. Nam, Radiation pressure acceleration of protons to $93 \mathrm{MeV}$ with circularly polarized petawatt laser pulses, Phys. Plasmas 23, 070701 (2016).

[9] F. Dollar, C. Zulick, A. Raymond, V. Chvykov, L. Willingale, V. Yanovsky, A. Maksimchuk, A. G. R. Thomas, and K. Krushelnick, Enhanced laser absorption from radiation pressure in intense laser plasma interactions, New J. Phys. 19, 063014 (2017).

[10] A. Henig, S. Steinke, M. Schnurer, T. Sokollik, R. Horlein, D. Kiefer, D. Jung, J. Schreiber, B. M. Hegelich, X. Q. Yan, J. Meyer-ter-Vehn, T. Tajima, P. V. Nickles, W. Sandner, and D. Habs, Radiation-Pressure Acceleration of Ion Beams Driven by Circularly Polarized Laser Pulses, Phys. Rev. Lett. 103, 245003 (2009).

[11] J. Denavit, Absorption of High-Intensity Subpicosecond Lasers on Solid Density Targets, Phys. Rev. Lett. 69, 3052 (1992).

[12] L. O. Silva, M. Marti, J. R. Davies, R. A. Fonseca, C. Ren, F. S. Tsung, and W. B. Mori, Proton Shock Acceleration in Laser-Plasma Interactions, Phys. Rev. Lett. 92, 015002 (2004).

[13] F. Fiuza, A. Stockem, E. Boella, R. A. Fonseca, L. O. Silva, D. Haberberger, S. Tochitsky, C. Gong, W. B. Mori, and C. Joshi, Laser-Driven Shock Acceleration of Monoenergetic Ion Beams, Phys. Rev. Lett. 109, 215001 (2012).

[14] T. Grismayer and P. Mora, Influence of a finite initial ion density gradient on plasma expansion into a vacuum, Phys. Plasmas 13, 032103 (2006). 
[15] D. Haberberger, S. Tochitsky, F. Fiuza, C. Gong, R. A. Fonseca, L. O. Silva, W. B. Mori, and C. Joshi, Collisionless shocks in laser-produced plasma generate monoenergetic high-energy proton beams, Nat. Phys. 8, 95 (2012).

[16] P. Antici, E. Boella, S. N. Chen, D. S. Andrews, M. Barberio, J. Bker, F. Cardelli, J. L. Feugeas, M. Glesser, P. Nicola, L. Romagnani, M. Scisci, M. Starodubtsev, O. Willi, J. C. Kieffer, V. Tikhonchuk, H. Ppin, L. O. Silva, E. d Humires, and J. Fuchs, Acceleration of collimated $45 \mathrm{MeV}$ protons by collisionless shocks driven in lowdensity, large-scale gradient plasmas by a $10^{20} \mathrm{~W} / \mathrm{cm}^{2}$, $1 \mu \mathrm{m}$ laser, Sci. Rep. 7, 16463 (2017).

[17] A. Pak, S. Kerr, N. Lemos, A. Link, P. Patel, F. Albert, L. Divol, B. B. Pollock, D. Haberberger, D. Froula, M. Gauthier, S. H. Glenzer, A. Longman, L. Manzoor, R. Fedosejevs, S. Tochitsky, C. Joshi, and F. Fiuza, Collisionless shock acceleration of narrow energy spread ion beams from mixed species plasmas using $1 \mu \mathrm{m}$ lasers, Phys. Rev. Accel. Beams 21, 103401 (2018).

[18] H. Zhang, B. F. Shen, W. P. Wang, S. H. Zhai, S. S. Li, X. M. Lu, J. F. Li, R. J. Xu, X. L. Wang, X. Y. Liang, Y. X. Leng, R. X. Li, and Z.Z. Xu, Collisionless Shock Acceleration of High-Flux Quasimonoenergetic Proton Beams Driven by Circularly Polarized Laser Pulses, Phys. Rev. Lett. 119, 164801 (2017).

[19] O. Tresca, N. P. Dover, N. Cook, C. Maharjan, M. N. Polyanskiy, Z. Najmudin, P. Shkolnikov, and I. Pogorelsky, Spectral Modification of Shock Accelerated Ions Using a Hydrodynamically Shaped Gas Target, Phys. Rev. Lett. 115, 094802 (2015).

[20] H. Zhang, B. F. Shen, W. P. Wang, Y. Xu, Y. Q. Liu, X. Y. Liang, Y. X. Leng, R. X. Li, X. Q. Yan, J. E. Chen, and Z.Z. Xu, Collisionless shocks driven by $800 \mathrm{~nm}$ laser pulses generate high-energy carbon ions, Phys. Plasmas 22, 013113 (2015).

[21] M. Liu, S. M. Weng, Y. T. Li, D. W. Yuan, M. Chen, P. Mulser, Z. M. Sheng, M. Murakami, L. L. Yu, X. L. Zheng, and J. Zhang, Collisionless electrostatic shock formation and ion acceleration in intense laser interactions with near critical density plasmas, Phys. Plasmas 23, 113103 (2016).

[22] T. D. Arber, K. Bennett, C. S. Brady, A. L. Douglas, M. G. Ramsay, N. J. Sircombe, P. Gillies, R. G. Evans, H. Schmitz, A. R. Bell, and C.P. Ridger, Contemporary particle-in-cell approach to laser-plasma modelling, Plasma Phys. Controlled Fusion 57, 113001 (2015).

[23] H. Karimabadi, N. Omidi, and K. Quest, Two-dimensional simulations of the ion/ion acoustic instability and electrostatic shocks, Geophys. Res. Lett. 18, 1813 (1991).

[24] G. Sarri, G. C. Murphy, M. E. Dieckmann, A. Bret, K. Quinn, I. Kourakis, M. Borghesi, L. Drury, and A.
Ynnerman, Two-dimensional particle-in-cell simulation of the expansion of a plasma into a rarefied medium, New J. Phys. 13, 073023 (2011).

[25] H. G. Rinderknecht, H. S. Park, J. S. Ross, P. A. Amendt, D. P. Higginson, S. C. Wilks, D. Haberberger, J. Katz, D. H. Froula, N. M. Hoffman, G. Kagan, B. D. Keenan, and E. L. Vold, Highly Resolved Measurements of a Developing Strong Collisional Plasma Shock, Phys. Rev. Lett. 120, 095001 (2018).

[26] W. S. Zhang, H. B. Cai, and S. P. Zhu, The formation and dissipation of electrostatic shock waves: the role of ion-ion acoustic instabilities, Plasma Phys. Controlled Fusion 60, 055001 (2018).

[27] M. King, R. J. Gray, H. W. Powell, R. Capdessus, and P. Mckenna, Energy exchange via multi-species streaming in laser-driven ion acceleration, Plasma Phys. Controlled Fusion 59, 014003 (2017).

[28] J. S. Ross, H.-S. Park, R. Berger, L. Divol, N. L. Kugland, W. Rozmus, D. Ryutov, and S. H. Glenzer, Collisionless Coupling of Ion and Electron Temperatures in Counterstreaming Plasma Flows, Phys. Rev. Lett. 110, 145005 (2013).

[29] T. N. Kato and H. Takabe, Electrostatic and electromagnetic instabilities associated with electrostatic shocks: Two-dimensional particle-in-cell simulation, Phys. Plasmas 17, 032114 (2010).

[30] Y. Ohira and F. Takahara, Oblique ion two-stream instability in the foot region of a collisionless shock, Astrophys. J. 688, 320 (2008).

[31] W. L. Kruer and K. G. Estabrook, J $\times$ B heating by very intense laser light, Phys. Fluids 28, 430 (1985).

[32] D. A. Tidman and N. A. Krall, Shock Waves in Collisionless Plasmas (Wiley-Interscience, New York, 1971).

[33] A. P. L. Robinson, D. Neely, P. McKenna, and R. G. Evans, Spectral control in proton acceleration with multiple laser pulses, Plasma Phys. Controlled Fusion 49, 373 (2007).

[34] P. Mora, Plasma Expansion into a Vacuum, Phys. Rev. Lett. 90, 185002 (2003).

[35] A. Grassi, L. Fedeli, A. Sgattoni, and A. Macchi, Vlasov simulation of laser-driven shock acceleration and ion turbulence, Plasma Phys. Controlled Fusion 58, 034021 (2016).

[36] K. D. Xiao, C. T. Zhou, K. Jiang, Y. C. Yang, R. Li, H. Zhang, B. Qiao, T. W. Huang, J. M. Cao, T. X. Cai, M. Y. Yu, S. C. Ruan, and X. T. He, High Z neoclassical transport: Application and limitation of analytical formulae for modelling JET experimental parameters, Phys. Plasmas 25, 012303 (2018). 